\title{
Adhesion, proliferation and osteogenic differentiation of mesenchymal stem cells in 3D printed poly- $\varepsilon$-caprolactone/hydroxyapatite scaffolds combined with bone marrow clots
}

\author{
PENGFEI ZHENG ${ }^{1-3^{*}}$, QINGQIANG YAO ${ }^{1,2^{*}}$, FENGYONG MAO ${ }^{2,3}$, \\ NANCY LIU ${ }^{4}$, YAN XU $^{1,2}$, BO WEI ${ }^{1,2}$ and LIMING WANG ${ }^{1,2}$ \\ ${ }^{1}$ Department of Orthopaedic Surgery, Nanjing First Hospital, Nanjing Medical University, Nanjing, Jiangsu 210006;
${ }^{2}$ Digital Medicine Institute, Nanjing Medical University, Nanjing, Jiangsu $210000 ;{ }^{3}$ Department of Orthopaedic
Surgery, Children's Hospital of Nanjing Medical University, Nanjing, Jiangsu 210008, P.R. China; ${ }^{4}$ Department of
Orthopaedic Research, Keck School of Medicine, University of Southern California, Los Angeles, CA 90033, USA
}

Received November 6, 2016; Accepted June 21, 2017

DOI: $10.3892 / \mathrm{mmr} .2017 .7266$

\begin{abstract}
Mesenchymal stem cells (MSCs), a stem cell population capable of multi-lineage differentiation, bound to porous biomaterial scaffolds, are widely used for bone tissue regeneration. However, there is evidence to suggest that MSC collection from bone marrow and expansion in vitro may result in phenotypic changes including a loss of differentiation potential and cell senescence. The aim of the present study was to find a facile and efficient approach to enable MSC adhesion and proliferation to scaffolds with osteogenic differentiation. Unprocessed bone marrow blood from the condyle of the distal femur in the rabbits were added to three-dimensional (3D) printed porous poly- $\varepsilon$-caprolactone/hydroxyapatite (PCL/HA) scaffolds with bone marrow clots (MC) formed, using two different methods for Group A (MC enriched scaffolds) and Group B (MC combined scaffolds), and then were cultured in osteogenic medium for 4 weeks. The scaffolds were assessed macroscopically and microscopically. Scaffold bioactivity and the proliferation and osteogenic differentiation of seeded MSCs were measured. Higher cellular viability and greater cell numbers in the scaffolds at later phases of culture were observed in Group B compared with Group A. In addition, Group B was associated with greater osteoinductivity, alkaline phosphatase activity and bony nodule formation, as assessed
\end{abstract}

Correspondence to: Professor Liming Wang, Department of Orthopaedic Surgery, Nanjing First Hospital, Nanjing Medical University, 68 Changle Road, Nanjing, Jiangsu 210006, P.R. China E-mail: limingwangnj@sina.com

\section{${ }^{*}$ Contributed equally}

Key words: mesenchymal stem cells, proliferation, 3D printing, scaffold, osteogenesis using scanning electron microscopy. Furthermore, reverse transcription-quantitative polymerase chain reaction analysis revealed that more osteogenic differentiation was present in Group B, compared with Group A. MC combined scaffolds proved to be a highly efficient, reliable and simple novel method for MSC adhesion, proliferation and differentiation. The MC combined PCL-HA multi-scale porosity scaffold may represent a candidate for future bone regeneration studies.

\section{Introduction}

Mesenchymal stem cells (MSCs) are of particular interest for therapeutic applications in tissue engineering, as they can differentiate into a number of lineages including chondrocytes, adipocytes and osteoblasts (1). MSCs exist in very low numbers in the highly cellular and heterogeneous bone marrow, and lack unique identifying markers necessary for definitive isolation. Standard protocols for the isolation of MSCs from the bone marrow include extraction of whole bone marrow, density gradient centrifugation and expansion of the mononuclear cell fraction on tissue culture polystyrene. While this protocol is well accepted for the use of MSCs, there is evidence to suggest that this expansion phase may result in phenotypic changes in MSCs, including a loss of differentiation potential and cell senescence $(2,3)$. The use of biomaterials for the incorporation and expansion of MSCs in a three-dimensional (3D) environment is of growing interest in the field, with several successful attempts to expand MSCs three-dimensionally with the use of micro carriers under dynamic conditions (4-6). However, these strategies do not eliminate the need for initial culture on plastic.

Marrow clots (MCs), formed by unprocessed bone marrow blood (UBMB) agglutination, provided a simple, convenient and relatively inexpensive biomimetic approach for tissue regeneration through MSC delivery and microenvironment modification inside 3D scaffolds $(7,8)$. Our previous study demonstrated that MCs enriched with scaffolds exhibit 
improved biological performance in inductive culture compared with conventional MSC-seeded scaffolds. However, the MC-mediated obstruction of pores can block the transfer of nutrients and prevent stem cell homing inside 3D printed (3DP) scaffolds, and during the culture of such scaffolds, MSCs were easily washed out when changing the medium (9). A facile and efficient MC concentration technique for 3DP scaffolds needs to be identified as an optimal procedure for tissue regeneration.

Previous studies have demonstrated that poly- $\varepsilon$-caprolactone (PCL), a biodegradable semi-crystalline linear aliphatic polyester, exhibits good mechanical properties (10). The Food and Drug Administration has approved this material for use as a drug delivery device and suture staple, and it has been extensively investigated as a biomaterial for regenerative medicine $(11,12)$. Hydroxyapatite (HA) has been widely investigated as an osteoconductive and inductive biomaterial for use as a porous bone substitute. PCL and HA have been blended to enhance cell proliferation and differentiation $(13,14)$. PCL-HA scaffolds with a precisely-controlled macroarchitecture and microstructure can be fabricated using a 3DP technique for bone regeneration $(15,16)$.

Furthermore, PCL-HA scaffolds produced by 3DP with suitable MC concentration may serve an important role in bone tissue engineering research. To address these issues, the present study involved preparation of 3DP PCL-HA scaffolds with two different $\mathrm{MC}$ concentration procedures, based on our previous findings (9). The MSC adhesion, proliferation and osteogenesis performance, cultured in osteogenic differentiation medium in vitro, was examined to evaluate the availability of MC concentration 3DP PCL-HA scaffolds for bone regeneration.

\section{Materials and methods}

Animals. Female New Zealand white rabbits $(n=10$; age, 5-6 months old; weight, 2.0-2.5 kg; Animal Core Facility, Nanjing Medical University, Nanjing, Jiangsu, China) were used for bone marrow blood collection using a micro-fracture procedure. Prior to the experiment, all rabbits were housed at room temperature $\left(25^{\circ} \mathrm{C}\right), 60 \%$ relative humidity and with a 12-h light/dark cycle for 1 week; all animals had free access to food and water. The use of animals in the present study was approved by the Institutional Animal Experiment Committee of Nanjing Medical University (Nanjing, China), and animals were treated according to the US National Institute of Health guidelines (National Institutes of Health, Bethesda, MA, USA). All animals underwent a veterinary examination to evaluate their general health status. All experimental procedures for bone marrow blood collection were performed under anesthesia with ear vein administration of $2 \%$ pentobarbital sodium (30 mg/kg; Sigma; Merck KGaA, Darmstadt, Germany).

Scaffold design and fabrication. 3DP PCL-HA porous scaffolds were designed and manufactured as detailed in the following section. The scaffolds were $4 \mathrm{~mm}$ in diameter, $2 \mathrm{~mm}$ in thickness, had $100 \%$ pore interconnectivity and a $500-\mu \mathrm{m}$ fiber diameter with a $0 / 45^{\circ} / 90^{\circ} / 135^{\circ}$ laydown pattern of fibers for porous structure following a previously published protocol (17). In brief, PCL powder (molecular weight $~ 60,000,3 \mathrm{D}$ Biotek,
LLC, North Brunswick, NJ, USA) and HA (Plasma Biotal Ltd., Buxton, UK) were dried separately for $24 \mathrm{~h}$ in a vacuum oven at $120^{\circ} \mathrm{C}$ and $40^{\circ} \mathrm{C}$, respectively. All chemicals used were of pharmaceutical grade, and the detailed characterization of these materials is previously described $(18,19)$. The scaffolds were fabricated using a $60 \%$ PCL/HA (by weight) (20) composite and a 3D fused deposition modelling (FDM) micro-fabrication technology (FDM 700 system; Nanjing Songsun Medical Technology Co, Ltd., Nanjing, Jiangsu, China) according to the manufacturer's protocol. Scaffolds ( $\mathrm{n}=80 /$ group) were fabricated with 10 sampled at each time point (day 1, weeks 1, 2 and 4 of in vitro culture; Fig. 1).

Preparation of MC concentration PCL-HA scaffold. A total of 10 rabbits were randomly divided into two groups $(n=5$ rabbits/group) and used for subsequent experiments. UBMB collection was performed using a micro-fracture procedure bilaterally on the condyle of the distal femur in the rabbits, as previously described (7). The amount of seeded MSCs was calculated following the protocol in our previous study $(7,21)$. For the MC enriched group (Group A), the scaffold was immersed and mixed in UBMB retrieved from one rabbit which contained heparin to prevent blood coagulation during the micro-fracture procedure. In brief, the 3DP scaffolds were immersed in UBMB for $1 \mathrm{~h}$ without agitation until they were fully enriched with $\mathrm{MC}$, and were then incubated at $37^{\circ} \mathrm{C}$ in a $5 \% \mathrm{CO}_{2}$ humidified incubator in L-Dulbecco's modified Eagle's medium (L-DMEM; Gibco; Thermo Fisher Scientific, Inc., Waltham, MA, USA) for 4 days, with the medium changed every 1-2 days until clear. For the MC combined group (Group B), the scaffold was plugged into the micro-fracture hole at the condyle of the distal femur until full of UBMB, and the MC formed. The scaffolds were then cultured in L-DMEM in the same manner as those in Group A (Fig. 2). Then two groups were cultured in osteogenic medium (Gibco; Thermo Fisher Scientific Inc.), supplemented with high-glucose DMEM, 10\% fetal bovine serum (both Gibco; Thermo Fisher Scientific, Inc.), $1 \%$ penicillin-streptomycin, $10 \mathrm{mmol} / \mathrm{l} \beta$-glycerophosphate, $50 \mu \mathrm{M}$ ascorbic acid and $100 \mathrm{nM}$ dexamethasone, for 28 days. A total of 10 samples were tested at each time point for each group at day 1 and at weeks 1, 2 and 4 of in vitro culture.

Macro and micro-morphology observation. The macro morphology of the cultured scaffolds was observed using an inverted microscope (IM) and a digital camera. The micro-structural morphology was observed using a scanning electron microscope (SEM; JEOL, Ltd., Tokyo, Japan), as in our previous study (9). In brief, specimens were fixed in $2.5 \%$ glutaraldehyde overnight, dehydrated using a series of graded ethanol solutions, dried overnight at room temperature and gold sputtered. SEM observation was then performed at an accelerating voltage of $5 \mathrm{keV}$.

Cell viability and proliferation assessment. Cell viability was assessed using a Live/Dead Reduced Biohazard Viability/Cytotoxicity kit (Molecular Probes; Thermo Fisher Scientific, Inc.) as in our previous study (9). Samples from each group were assessed at day 1, 14 and 28. In brief, samples were washed using phosphate buffered saline, incubated in the 


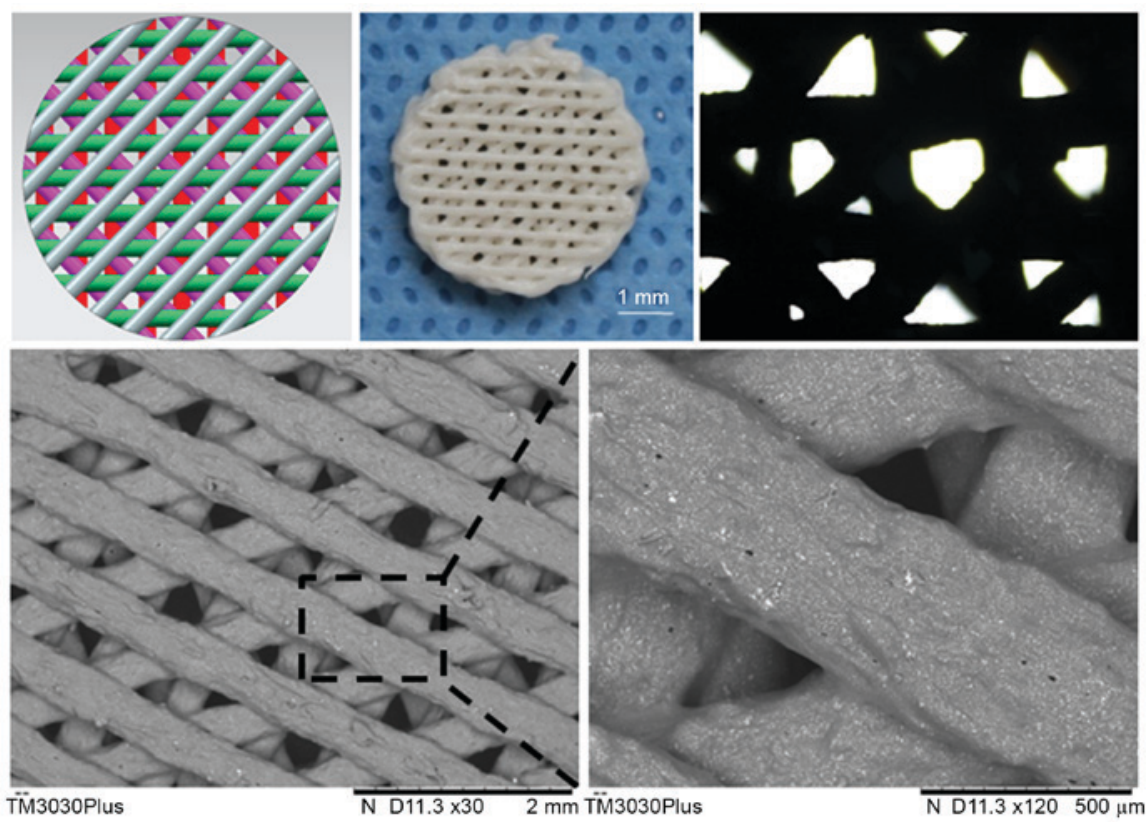

Figure 1. Manufacturing a 3D PCL-HA porous scaffold. The fabricated PCL-HA scaffolds matched the design parameters established using CAD. The 3D fiber porous structure as observed using an inverted microscope and a scanning electron microscope. PCL-HA, poly- $\varepsilon$-caprolactone-hydroxyapatite.
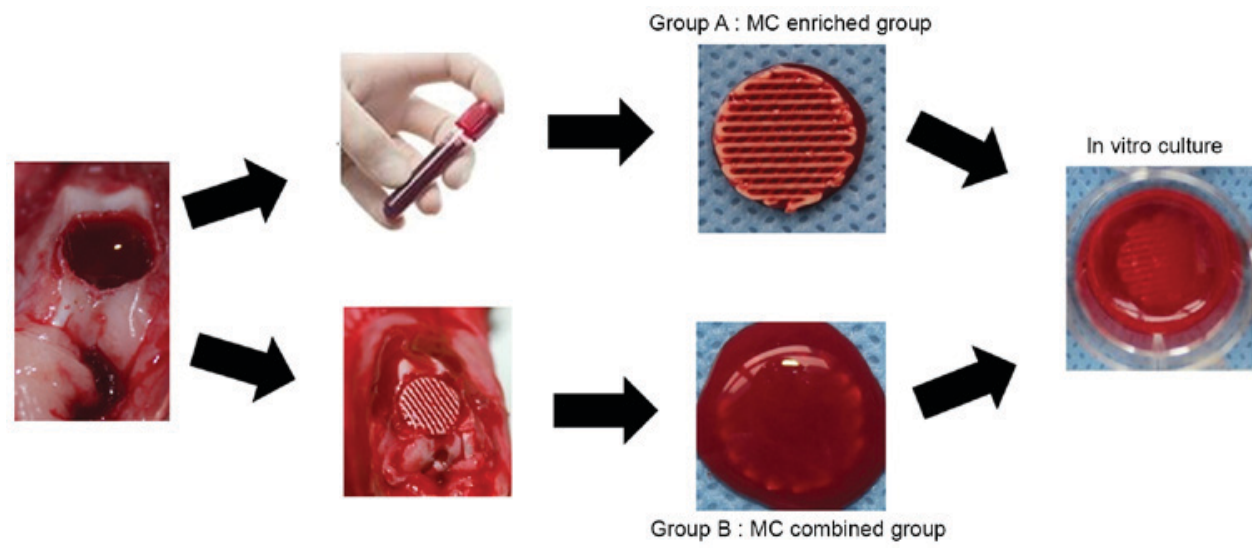

Figure 2. Marrow clots blended with a poly- $\varepsilon$-caprolactone/hydroxyapatite scaffold using two different procedures. In Group A, the scaffold was immersed in unprocessed bone marrow blood for $1 \mathrm{~h}$ without agitation until $\mathrm{MC}$ were obtained in each structure. In Group B, the scaffold was plugged into a micro-fracture hole at the condyle of the distal femur until full with unprocessed bone marrow blood and a MC formed. The scaffolds were cultured in vitro and 10 samples were analyzed at day 1 , and 1,2 and 4-week time-points. MC, marrow clots.

dilute dye solution for $15 \mathrm{~min}$ in the dark at room temperature and then fixed in $4 \%$ glutaraldehyde for $1 \mathrm{~h}$. These samples were observed and imaged using a confocal microscope (Leica T7100M Confocal Microscope, Leica Microsystems $\mathrm{GmbH}$, Wetzlar, Germany). Live cells were stained green and dead cells were stained red.

A MTT cell proliferation assay kit (Roche Applied Science, Penzburg, Germany) was used to assess proliferation of the cells on the scaffolds, following the protocol supplied by the manufacturer. After 1, 7, 14 and 28 days of cell culture, $20 \mu \mathrm{l}$ MTT reagent was added to each well of the microtiter plates containing the scaffolds, and cells were incubated for $4 \mathrm{~h}$ at $37^{\circ} \mathrm{C}$. After $200 \mu \mathrm{l}$ solubilization solution (DMSO; Roche Diagnostics, Indianapolis, IN, USA) was added to each well, the plates were incubated overnight. The absorbance was measured at $595 \mathrm{~nm}$ using a microplate reader (Bio-Rad Laboratories, Inc., Hercules, CA, USA).
Osteogenic differentiation analyses of the scaffolds. The intracellular alkaline phosphatase (ALP) activity on the cultured scaffolds was compared between the two groups on days $0,7,14$ and 28 to estimate cell differentiation. Cell lysates were tested for ALP activity using a SensoLyte ${ }^{\mathrm{TM}}$ pNPP Alkaline Phosphatase Assay kit (AnaSpec, Fremont, CA, USA) according the manufacturer's protocol. The absorbance was measured at $415 \mathrm{~nm}$ using a microplate reader (Bio-Rad Laboratories, Inc.) (22).

Reverse transcription-quantitative polymerase chain reaction (RT-qPCR). The expression levels of osteogenic genes on days 14 and 28 were measured using RT-qPCR. The scaffolds were lysed using TRIzol (Invitrogen; Thermo Fisher Scientific, Inc.). The samples in TRIzol were incubated at $-80^{\circ} \mathrm{C}$ until RNA isolation. Total RNA was isolated and reverse transcribed to cDNA using a High-Capacity cDNA Reverse Transcription 
Table I. Primer nucleotide sequences for RT-PCR.

\begin{tabular}{|c|c|}
\hline Gene & Primer nucleotide sequence \\
\hline GAPDH & $\begin{array}{l}\text { Forward: 5-GCTTTGCCCCGCGATCTAATG } \\
\text { TTC-3 } \\
\text { Reverse: 3-GCCAAATCCGTTCACTCCGAC } \\
\text { CTT-5 }\end{array}$ \\
\hline $\operatorname{Cbf} \alpha 1$ & $\begin{array}{l}\text { Forward: 5-GAGGGCCACAAGTTCTATCT } \\
\text { GGA-3 } \\
\text { Reverse: 3-GGTGGTCCGCGATGATCTC-5 }\end{array}$ \\
\hline $\mathrm{OCN}$ & $\begin{array}{l}\text { Forward: 5-ATGAGAGCCCTCACACTCCTC-3 } \\
\text { Reverse: 3-GCCGTAGAAGCGCCGATAGGC-5 }\end{array}$ \\
\hline OPN & $\begin{array}{l}\text { Forward: 5-TTAGGGGACCCAGAGATGC-3 } \\
\text { Reverse: 3-AGATGTGTCATGAGGTTTGTGC-5 }\end{array}$ \\
\hline
\end{tabular}

kit (Applied Biosystems; Thermo Fisher Scientific, Inc.). qPCR was performed for the quantification of gene expression using the primers listed in Table I in an ABI Prism 7500 sequence detection system (Applied Biosystems; Thermo Fisher Scientific, Inc.). The target genes osteopontin (OPN), osteocalcin (OCN) and core binding factor $\alpha 1$ (Cbf $\alpha 1)$, were normalized against GAPDH expression. Each $20 \mu \mathrm{l}$ reaction mix contained $10 \mu 12 \mathrm{X}$ LightCycler $^{\circledR} 480$ SYBR Green I Master (Roche Diagnostics, Basel, Switzerland), $10 \mathrm{ng}$ modified DNA and $0.8 \mu \mathrm{l}$ primer mix $(10 \mathrm{pM} / \mu \mathrm{l})$. qPCR was performed using a LightCycler ${ }^{\circledR} 480$ with the following cycling conditions: $95^{\circ} \mathrm{C}$ of initial denaturation for $10 \mathrm{~min}$ followed by 40 cycles of denaturation at $95^{\circ} \mathrm{C}$ for $15 \mathrm{sec}$, annealing at $60^{\circ} \mathrm{C}$ for $1 \mathrm{~min}$, annealing at $62^{\circ} \mathrm{C}$ for $10 \mathrm{sec}$, then polymerization at $72^{\circ} \mathrm{C}$ for $20 \mathrm{sec}$. A melting curve was created by cooling the products at $50^{\circ} \mathrm{C}$ for $30 \mathrm{sec}$ and then heating to $80^{\circ} \mathrm{C}$ at a rate of $0.1^{\circ} \mathrm{C} / \mathrm{sec}$, while simultaneously measuring the fluorescence. Data were analyzed via the comparative quantitation cycle (Cq) method (23). $\Delta \mathrm{Cq}$ values were first calculated using the formula: $\Delta \mathrm{Cq}=\mathrm{Cq}_{\text {Target gene }}-\mathrm{Cq}_{\mathrm{GAPDH}}$; then the mean level of target gene $\left(2^{-\mathrm{ACq}}\right)$ in all samples was determined.

Histology staining. For Alizarin Red staining, cells on scaffolds were fixed using $3.7 \%$ formaldehyde for $24 \mathrm{~h}$ at $37^{\circ} \mathrm{C}$ and then stained with Alizarin Red S (Nanjing KeygGen Biotech Co., Ltd., Nanjing, China) for $15 \mathrm{~min}$ at $25^{\circ} \mathrm{C}$. Scaffolds were washed five times using deionized water to remove excess stain and then air-dried. The morphology of the stained scaffolds was assessed using a digital camera.

Statistical analysis. All statistical analysis was performed using SPSS 13.0 statistical software (SPSS Inc., Chicago, IL, USA). Data are presented as the mean \pm standard deviation. The difference between the groups was analyzed by one-way analysis of variance and a Student-Newman-Keuls post hoc test. $\mathrm{P}<0.05$ was considered to indicate a statistically significant difference.

\section{Results}

Scaffold characterization. The fabricated PCL-HA scaffolds, a $100 \%$ pore interconnectivity and $500 \mu \mathrm{m}$ fiber diameter with a $0 / 45^{\circ} / 90^{\circ} / 135^{\circ}$ laydown pattern of fibers for porous structure cylinder with a 4-mm diameter and a 2-mm height, matched the design parameters as assessed through IM and SEM observations. The gross scaffold morphology and cross sectional view of the composite are presented in Fig. 1.

Observation of macro and micro-structure of the scaffold. SEM imaging revealed that the pores of the scaffolds were filled and obstructed by fibroblast-like tissue, monocytes and erythrocytes at day 1 in Groups A and B (Fig. 3). Further observation of the scaffold morphology revealed that most erythrocytes and UBMB matrix inside the scaffold pores were washed off after 1 week in normal culture medium in both groups. There was a decrease in the red coloration of the scaffolds, which was more pronounced in scaffolds in Group A. As time progressed, a gradual accumulation of extracellular matrix (ECM) was observed in both groups. Meanwhile, the density of the scaffold content gradually increased with a subsequent increase of the matrix in the pores during the culture period, as assessed using IM observations (data not shown).

Cell adhesion and proliferation. A cell viability assessment using confocal imaging of live/dead assay samples demonstrated that green-stained live cells in Group B were apparently increased at the 4-week in vitro time-point, compared with those in Group A. The pore obstruction and recanalization could also be observed in the confocal images (Fig. 4A). Cell proliferation on the scaffolds was assessed using an MTT assay (Fig. 4B). The optical density (OD) values were similar in the two groups during the first 14 days of culture. At day 28, the OD values were higher in Group B. The ratio of living cells was the same as the MTT values. Therefore, the MC combined scaffold was considered to be more effective for cell adhesion and proliferation.

Biochemical analyses of the scaffolds. ALP activity was measured to examine the osteogenic differentiation of MSCs on the scaffolds (Fig. 5A). ALP activity gradually increased with time. The ALP activity of Group B was significantly greater compared with Group A, compared at day $28(\mathrm{P}<0.05)$. SEM images of the scaffolds at day 28 (Fig. 5B) demonstrate higher mineralization in the Group B scaffolds, which corroborates with the ALP activity results. The expression levels of osteogenic genes on days 14 and 28 were measured using RT-qPCR (Fig. 5C). Group B demonstrated a higher mean expression of Cbf $\alpha 1$ compared with Group A on day 14. However, Cbf $\alpha 1$ expression in Group B on day 28 was lower than in Group A. The mRNA expression levels of OPN and OCN in both Group $A$ and $B$ gradually increased during in vitro culture, with the level of expression in Group B being significantly higher than in Group A (Fig. 5C; $\mathrm{P}<0.05$ ).

Alizarin Red $S$ staining. When cultured in the osteogenic supplements, MSCs in both groups calcified their matrix by day 28 (Fig. 5D). Calcification is a standard marker for osteogenesis and osteogenic differentiation, and can be assessed using Alizarin Red S staining. The staining in Group B was visibly darker than in Group A. Controls using empty scaffolds without MSCs did not mineralize, indicating that the 

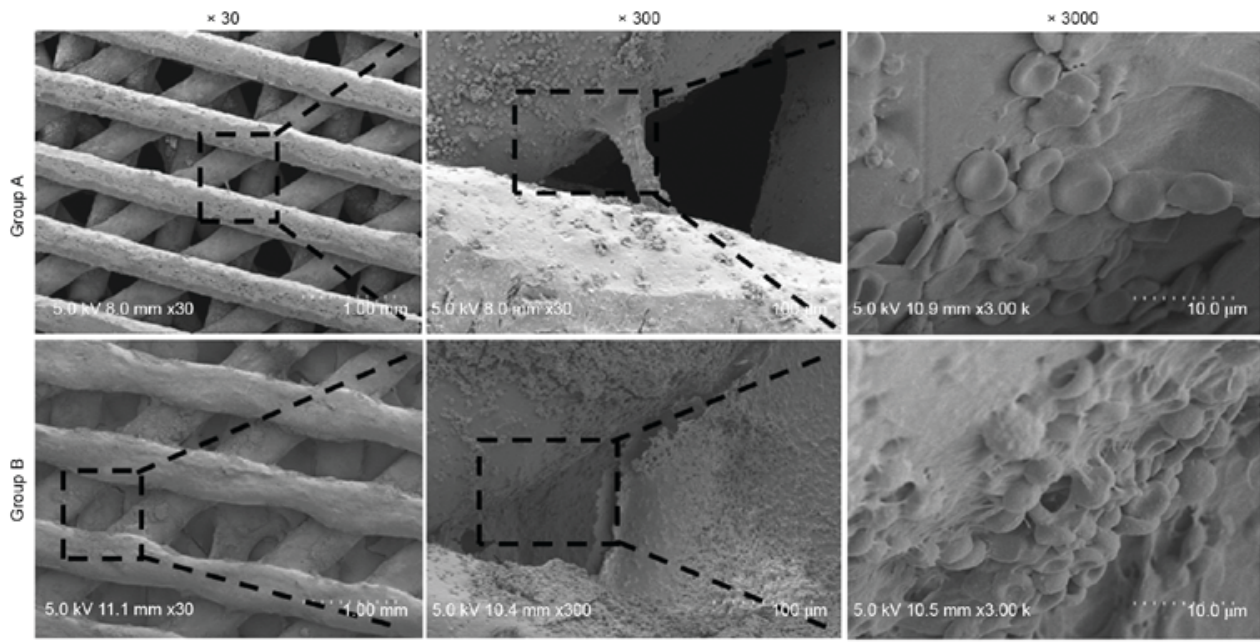

Figure 3. Micro-morphology of the composite scaffolds at day 1 as assessed using a SEM. A SEM confirmed a greater amount of fibroblast-like tissue, monocytes and erythrocytes inside the pores of Group B scaffolds, compared with Group A scaffolds. SEM, standard electron microscope.
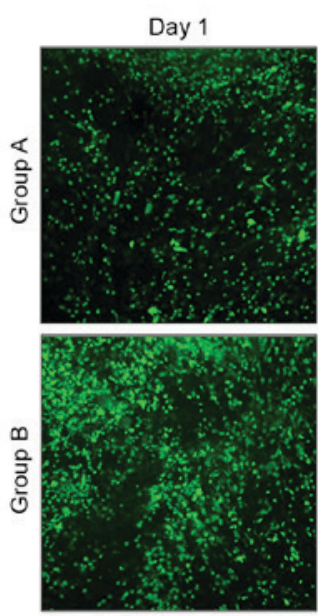

Day 14
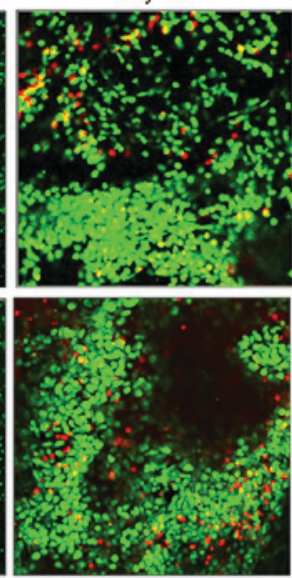

Day 28
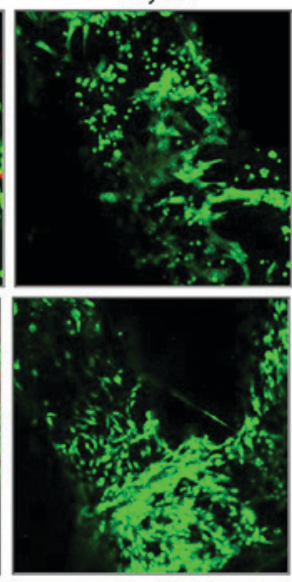
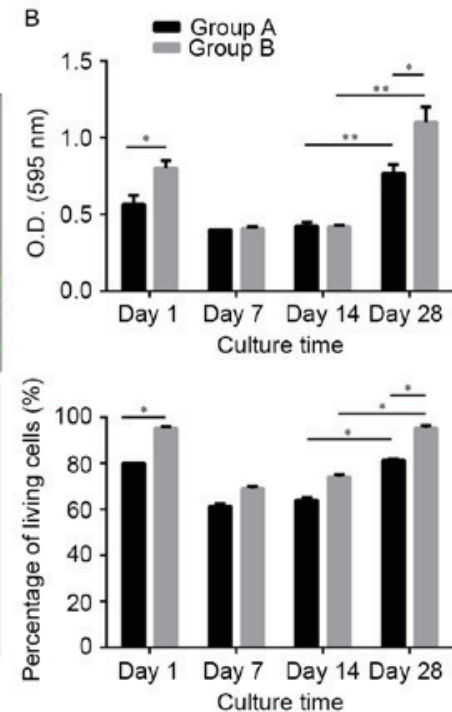

Figure 4. Live/dead cell assay of cells seeded on the scaffolds. (A) Representative confocal micrographs of live (green) and dead (red) cells in Groups A and B. (B) The ratio of live/dead cells in the two groups decreased at week 1 and increased after 2 weeks in culture, and this tendency was more pronounced in Group B. Data are presented as the mean \pm standard deviation. ${ }^{*} \mathrm{P}<0.05,{ }^{* *} \mathrm{P}<0.01$. O.D., optical density.

mineralization was mediated by the MSCs and was not a result of non-specific calcium precipitation.

\section{Discussion}

Bone marrow cell systems include both non-hematopoietic stromal cells and hematopoietic cells. The stromal functions as a scaffold, composed of a network of cells that provide physical and functional support to the hematopoietic cells. The stromal fraction, characteristically, is able to adhere to tissue culture plastic, while the non-adherent hematopoietic cells can be readily removed from the adherent stromal cell cultures using a simple wash step (24). From the SEM and live/dead confocal images of the two groups, the present study demonstrated that the pores of the scaffolds were filled with cells and erythrocytes from the MC at day 1. During the following culture, erythrocytes and other non-adherent hematopoietic cells were gradually eluted. This may have caused the red color to fade and the initially observed decrease in the live/dead cell ratio. An increase in the live/dead cell ratio was observed in both groups at the 2-week time-point, with MSC proliferation. However, in the MC combined PCL-HA scaffolds, gradual cell proliferation was observed over time, which may have caused a higher live/dead cell ratio and a lower cell/ECM ratio during culture, compared with Group A.

The present study compared two different methods of MC concentration by observing cell adhesion and proliferation. The results demonstrated more stem cell adhesion and proliferation and improved osteogenic differentiation properties in the scaffold combined with UBMB. In the MC-enriched scaffold group, heparin was used to interfere with the blood clotting process during the micro-fracture procedure. Ultimately, heparin prevented the conversion of fibrinogen into fibrin, resulting in preventing blood coagulation. However, when 


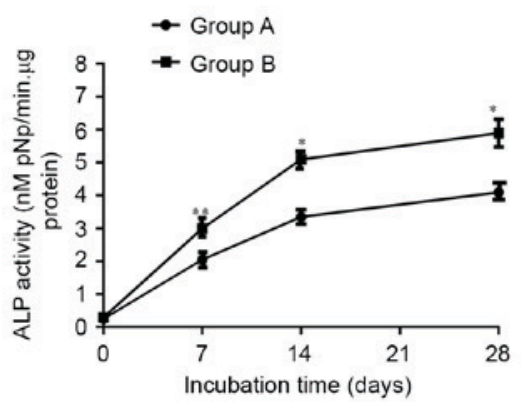

C

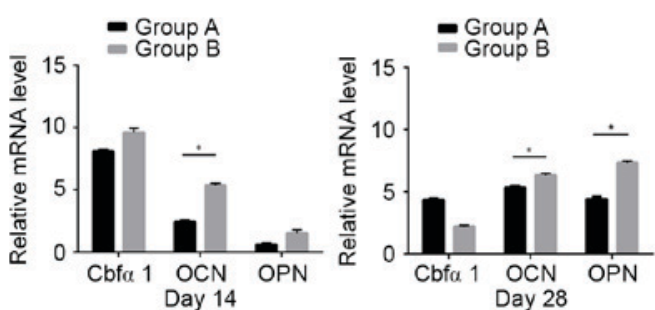

B

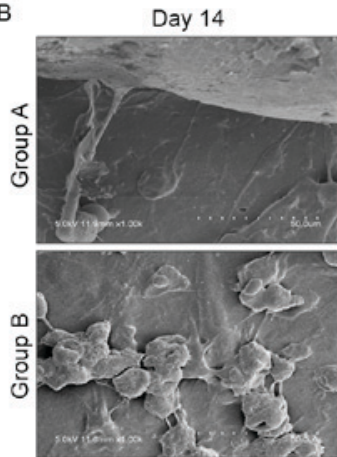

$\mathrm{D}$

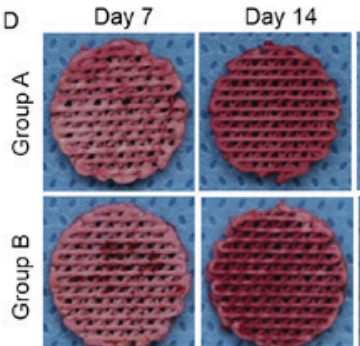

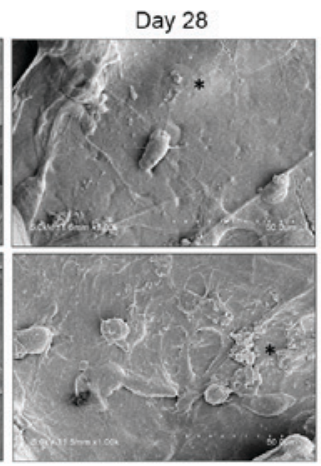

Day 28

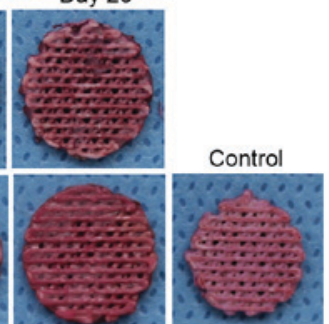

Figure 5. Biochemical and gene expression assays of the cultured scaffolds. (A) ALP activity. ${ }^{*} \mathrm{P}<0.05,{ }^{* *} \mathrm{P}<0.01$. (B) Scanning electron microscope images of cellular scaffolds in Groups A and B at days 14 and 28. (C) The gene expression levels of OPN, OCN and Cbfal were normalized to the housekeeping gene GAPDH and compared at days 14 and day 28. "P<0.05. (D) Alizarin Red S staining of the scaffolds in both groups. The control was an untreated scaffold also stained with Alizarin Red S. Data are expressed as the mean \pm standard deviation. OPN, osteopontin; OCN, osteocalcin; Cbf $\alpha 1$, core binding factor $\alpha 1$; ALP, alkaline phosphatase.

clotting was not impaired, the fibrin formed bridge shaped structures both along the rods and across the pores of the scaffolds combined with BMB clots, thereby promoting cell adhesion in these scaffolds.

Previous investigations of 3D fabricated porous scaffolds have demonstrated that the formation of new tissues is greatly influenced by the pore size, porosity and the 3D structure of the scaffold $(11,25)$. Synthetic scaffolds for bone regeneration must be a 3D structure with interconnected porosity and appropriate pore sizes to assure good vascularization, nutrient delivery and cell attachment in growth (26-28). A large pore size is necessary to deliver a sufficient number of cells, and interconnected porosity is required for the easy diffusion of nutrients. Previous studies have suggested that a small pore size improves bone growth into scaffolds and provides attachment points for osteoblasts (29). The present study fabricated a PCL/HA scaffold with macro and micro porosity for osteogenic differentiation.

In the present study, the ALP activity in Group B was significantly increased compared with Group A. Similarly, RT-qPCR demonstrated that that the expression of OCN and OPN in Group B was higher than in Group A. Cbf $\alpha 1$ expression was higher in Group B at day 14, but the expression of Cbf $\alpha 1$ in the two groups gradually decreased over time, and the expression in Group A was slightly higher than in Group $\mathrm{B}$ at day 28. Cbfa1 inhibits the transition of osteoblasts to osteocytes and thereby maintains osteoblastic cells at an immature stage and effectively directs the formation of immature bone. Normally, the expression of $\mathrm{Cbf} \alpha 1$ is first detected in pre-osteoblasts and is upregulated in immature osteoblasts, but downregulated in mature osteoblasts $(30,31)$. The present study demonstrated that Group B exhibited a greater osteogenetic capacity than Group A. This may be because the UBMB concentration method used in Group B had a significant effect on cell adhesion and osteogenesis compared with Group A, which was demonstrated by the cell proliferation assay and the Alizarin Red S staining. Perhaps the different concentration of growth factors in the MC also attribute to the osteogenetic difference, which requires checking in future studies.

There are some limitations to the present study. The difference between the two different coagulation methods in UBMB was not determined. Whether there is a significant change in the blood composition of the bone marrow after the addition of heparin needs to be clarified by further research. Although MC-PCL-HA demonstrated good osteogenic properties in vitro, the performance of the MC-PCL-HA scaffolds in autologous in vivo studies requires further investigation. The composition of the UBMB is complex. Though most of the non-adherent cells were eluted in culture, residual cellular constituents remained in the pores of the scaffold, which is much closer to the autogenous bone repair process. Furthermore, as an autologous procedure, MC may circumvent the barriers encountered in allograft procedures, including immune rejection, pathogen transmission, issues with packaging, storage and shipping, and difficulties in clinical adoption.

In conclusion, MC-combined scaffolds proved to be a highly efficient, reliable and simple novel method for MSC adhesion, proliferation and osteogenic differentiation. The MC-combined PCL-HA multi-scale porosity scaffold can be a candidate for future bone regeneration studies.

\section{Acknowledgements}

The present study was supported by the National Natural Science Foundation of China (grant no. 81171745) and the Nanjing Medical Science and Technology Development Foundation (grant no. YKK15132). 


\section{References}

1. Prockop DJ: Marrow stromal cells as stem cells for nonhematopoietic tissues. Science 276: 71-74, 1997.

2. Baksh D, Song L and Tuan RS: Adult mesenchymal stem cells: Characterization, differentiation and application in cell and gene therapy. J Cell Mol Med 8: 301-316, 2004.

3. Bara JJ, Richards RG, Alini M and Stoddart MJ: Concise review: Bone marrow-derived mesenchymal stem cells change phenotype following in vitro culture: Implications for basic research and the clinic. Stem Cells 32: 1713-1723, 2014.

4. Goh TK, Zhang ZY, Chen AK, Reuveny S, Choolani M, Chan JK and Oh SK: Microcarrier culture for efficient expansion and osteogenic differentiation of human fetal mesenchymal stem cells. Biores Open Access 2: 84-97, 2013.

5. Santos FD, Andrade PZ, Abecasis MM, Gimble JM, Chase LG, Campbell AM, Boucher S, Vemuri MC, Silva CL and Cabral JM: Toward a clinical-grade expansion of mesenchymal stem cells from human sources: A microcarrier-based culture system under xeno-free conditions. Tissue Eng Part C Methods 17: 1201-1210, 2011.

6. Hervy M, Weber JL, Pecheul M, Dolley-Sonneville P, Henry D, Zhou Y and Melkoumian Z: Long term expansion of bone marrow-derived hMSCs on novel synthetic microcarriers in xeno-free, defined conditions. PLoS One 9: e92120, 2014

7. Wei B, Jin C, Xu Y, Du X, Yan C, Tang C, Ansari M and Wang L: Chondrogenic differentiation of marrow clots after microfracture with BMSC-derived ECM scaffold in vitro. Tissue Eng Part A 20: 2646-2655, 2014.

8. Rohner D, Hutmacher DW, Cheng TK, Oberholzer M and Hammer B: In vivo efficacy of bone-marrow-coated polycaprolactone scaffolds for the reconstruction of orbital defects in the pig. J Biomed Mater Res B Appl Biomater 66: 574-580, 2003.

9. Yao QQ, Wei B, Liu N, Li C, Guo Y, Shamie AN, Chen J, Tang C, Jin CZ, Xu L, et al: Chondrogenic regeneration using bone marrow clots and a porous polycaprolactone-hydroxyapatite scaffold by three-dimensional printing. Tissue Eng Part A 21: 1388-1397, 2015

10. Chen M, Parsons AJ, Felfel RM, Rudd CD, Irvine DJ and Ahmed I: In-situ polymerisation of fully bioresorbable polycaprolactone/phosphate glass fibre composites: In vitro degradation and mechanical properties. J Mech Behav Biomed Mater 59: 78-89, 2016.

11. Coombes AG, Rizzi SC, Williamson M, Barralet JE, Downer S and Wallance WA: Precipitation casting of polycaprolactone for applications in tissue engineering and drug delivery. Biomaterials 25: 315-325, 2004.

12. Carletti E, Motta A and Migliaresi C: Scaffolds for tissue engineering and 3D cell culture. Methods Mol Biol 695: 17-39, 2011.

13. Park SA, Lee SH and Kim WD: Fabrication of porous polycaprolactone/hydroxyapatite (PCL/HA) blend scaffolds using a 3D plotting system for bone tissue engineering. Bioprocess Biosyst Eng 34: 505-513, 2011.

14. Hollister SJ: Porous scaffold design for tissue engineering. Nat Mater 4: 518-524, 2005.

15. Basile MA, d'Ayala GG, Malinconico M,Laurienzo P, Coudane J, Nottelet B, Raqione FD and Oliva A: Functionalized PCL/HA nanocomposites as microporous membranes for bone regeneration. Mater Sci Eng C Mater Biol Appl 48: 457-468, 2015.

16. Hutmacher DW, Schantz T, Zein I, Nq KW, Teoh SH and Tan KC: Mechanical properties and cell cultural response of polycaprolactone scaffolds designed and fabricated via fused deposition modeling. J Biomed Mater Res 55: 203-216, 2001.
17. Yao QQ, Wei B, Guo Y, Jin C, Du X, Yan C, Yan J, Hu W, $\mathrm{Xu} Y$, Zhou Z, et al: Design construction and mechanical testing of digital 3D anatomical data-based PCL-HA bone tissue engineering scaffold. J Mater Sci Mater Med 26: $5360,2015$.

18. Li LH, Kommareddy KP, Pilz C, Zhou CR, Fratzl P and Manjubala I: In vitro bioactivity of bioresorbable porous polymeric scaffolds incorporating hydroxyapatite microspheres. Acta Biomater 6: 2525-2531, 2010 .

19. Sun H, Mei L, Song C, Cui X and Wang P: The in vivo degradation, absorption and excretion of PCL-based implant. Biomaterials 27: 1735-1740, 2006.

20. Ding C, Qiao Z, Jiang W, Li H, Wei J, Zhou G and Dai K: Regeneration of a goat femoral head using a tissue-specific, biphasic scaffold fabricated with CAD/CAM technology. Biomaterials 34: 6706-6716, 2013.

21. Tang C, Xu Y, Jin C, Min BH, Li Z, Pei X and Wang L: Feasibility of autologous bone marrow mesenchymal stem cell-derived extracellular matrix scaffold for cartilage tissue engineering. Artif Organs 37: E179-E190, 2013.

22. Park SA, Lee SH and Kim WD: Fabrication of porous polycaprolactone/hydroxyapatite (PCL/HA) blend scaffolds using a 3D plotting system for bone tissue engineering. Bioprocess Biosyst Eng 34: 505-513, 2011

23. Livak KJ and Schmittgen TD: Analysis of relative gene expression data using real-time quantitative PCR and the 2(-Delta Delta C(T)) method. Methods 25: 402-408, 2001.

24. Black CR, Goriainov V, Gibbs D, Kanczler J, Tare RS and Oreffo RO: Bone tissue engineering. Curr Mol Bio Rep 1: 132-140, 2015.

25. Woodfield TB, Malda J, de Wijn J, Péters F, Riesle J and van Blitterswijk CA: Design of porous scaffolds for cartilage tissue engineering using a three-dimensional fiber-deposition technique. Biomaterials 25: 4149-4161, 2004.

26. Hollister SJ: Porous scaffold design for tissue engineering. Nat Mater 4: 518-524, 2005.

27. Woodard JR, Hillldore AJ, Lan SK, Park CJ, Morgan AW, Eurell JA, Clark SG, Wheeler MB, Jamison RD and Waqoner Johnson AJ: The mechanical properties and osteoconductivity of hydroxyapatite bone scaffolds with multi-scale porosity. Biomaterials 28: 45-54, 2007.

28. Mankani MH, Afghani S, Franco J, Launey M, Marshall S, Marshall GW, Nissenson R, Lee J, Tomisa AP and Saiz E: Lamellar spacing in cuboid hydroxyapatite scaffolds regulates bone formation by human bone marrow stromal cells. Tissue Eng Part A 17: 1615-1623, 2011.

29. Bignon A, Chouteau J, Chevalier J, Fantozzi G, Carret JP, Chavassieux P, Boivin G, Melin M and Hartmann D: Effect of micro- and macroporosity of bone substitutes on their mechanical properties and cellular response. J Mater Sci Mater Med 14: 1089-1097, 2003.

30. Battula VL, Le PM, Sun J, Queen TM, Somanchi A, Wang RY, Strunk D, Shpall EJ, Ruvolo P, Kantarjian H, et al: Mesenchymal stem cells differentiate into osteoblasts in the presence of AML cells through up-regulation of RUNX2 and induce chemo-resistance. Cl Lymph Myelom Le 15: S185-S186, 2015.

31. Stein GS, Lian JB, van Wijnen AJ, Stein JL, Montecino M, Javed A, Zaidi SK, Young DW, Choi JY and Pockwinse SM: Runx 2 control of organization, assembly and activity of the regulatory machinery for skeletal gene expression. Oncogene 23: 4315-4329, 2004. 